MS32-P16

\section{Guest loading into highly flexible MOF pores driven by molecular recognition: a stepwise SCXRD investigation}

Claudia Carraro $^{1}$, Davide Balestri ${ }^{1}$, Paolo Pio Mazzeo ${ }^{2}$, Paolo

Pelagatti ${ }^{1}$, Nicola Demitri ${ }^{3}$, Alessia Bacchi ${ }^{2}$

1. University of Parma, Parma, Italy

2. University of Parma, Centro Interdipartimentale Biopharmanet-

TEC, Parma, Italy

3. Sincrotrone Elettra, Trieste, Italy

email: claudia.carraro@studenti.unipr.it

MOFs (Metal Organic Frameworks) are a large class of ultraporous crystalline polymers deriving from the self-assembly of metallic ions or clusters with polytopic organic ligands. The high modularity of their synthesis allows a fine tuning of their porosity, making possible the confinement of molecular species of different shape and size.

Starting from the pioneeristic concept of crystalline-sponge method [1], we here propose a systematic way to embed small molecular aggregates inside home-made porous crystalline materials, with the aim of exploring the structural aspects of nanoconfinement and stabilizing the guest molecules inside the cavities of the structure. The challenge of this idea stands in the possibility to neatly "freeze", within a crystal, ordered supramolecular clusters of molecules that would form a liquid in their natural state at ambient conditions, and visualize their supramolecular aggregation, at different loading time.

In particular, we propose an unprecedent stepwise characterization of the evolution of the guest loading into metal-organic framework driven by host-guest interactions , focusing on the description of supramolecular aggregate of the guests inside the pores guided by guest-guest interactions. The MOF crystals were soaked for different time into the pure liquid guest and sequentially characterized via SCXRD.

The guest we focused on is eugenol, which is a volatile phenolic constituent of clove essential oil obtained from Eugenia caryophyllata buds and leaves. It is a functional ingredient of numerous products which have been used in the pharmaceutical, agro-food and cosmetic industry. The wide range of eugenol activities derived from it antimicrobial, anti-inflammatory, analgesic and antioxidant properties.

References:

[1] Y. Inokuma, S. Yoshioka, J. Ariyoshi, T. Arai, Y. Hitora, K. Takada, S. Matsunaga, K.Rissanen and M. Fujita, 2013, Nature, 495, 461-466.

Keywords: MOF, molecular recognition

\section{MS32-P17}

\section{Synthetic strategies for delivering targeted supramolecular topologies of crystalline metal-containing solids}

Marijana Đaković ${ }^{1}$, Ivan Kodrin ${ }^{1}$, Christer B. Aakeröy², Mladen Borovina

1. Department of Chemistry, Faculty of Science, University of Zagreb, Zagreb, Croatia

2. Department of Chemistry, Kansas State University, Manhattan, United States of America

email: mdjakovic@chem.pmf.hrP

ractical crystal engineering, as a bottom-up approach for building-up functional materials, is transitioning from fundamental explorations to more targeted applications. This is illustrated by the fact that numerous productive strategies for non-covalent synthesis of organic solids-state systems have been reported.[1] In contrast, reliable synthetic protocols for the assembly of metal-containing systems, especially those that employ a combination of coordinate-covalent and relatively weak and readily reversible hydrogen and/or halogen bonds, have not received anywhere near the same attention.

To develop robust synthetic protocols for assembling metal-organic crystalline solids in a preconceived manner, we have focused our attention on several functionalities that are known to form robust and reliable supramolecular synthons in organic surrounding and examine their potential transferability to metal-organic systems. Coordination complexes often present additional challenges to the supramolecular chemist as they frequently contain functionalities and binding sites that can interfere with the intended crystal engineering strategy.

Here, we have carried out a systematic structural study of a number of classes of metal(II) complexes with pyridine-type and charge-balancing ligands, and rationalized their supramolecular behaviour against a back-drop of molecular electrostatic potential (MEP) surface values. Based on our extensive experimental data,[2-5] we are able to address several questions of critical importance to the successful assembly of metal-containing systems: (a) Under what conditions can we simply transfer specific synthons from organic to metal-organic solid-state systems? (b) Can a simplified electrostatic view of non-covalent interactions and the results from the calculated MEP values be used to direct and rationalize supramolecular synthesis? and (c) How can we derive supramolecular synthetic protocols in much the same way as classical synthesis is systematically altered and refined in response to product yields?

References:

1. Aakeröy, C.B. \& Sinha, A.S., Co-crystals: Preparation,

Characterization and Applications, Monographs in Supramolecular Chemsitry, RSC, 2018.

2. Kukovec, B.M., Malik, M., Kodrin, I., Aakeröy, C.B. \& Đaković,

M. (2016). Cryst. Growth Des., 16, 7308-7317.

3. Borovina, M., Kodrin, I. \& Đaković, M. (2018). CrystEngComm,

20, 539-549.

4. Đaković, M., Soldin, Ž., Kukovec, B.-M., Kodrin, I., Aakeröy,

C.B., Baus, N. \& Rinkovec, T. (2018). IUCrJ 5, 13-21.

5. Borovina, M., Kodrin, I. \& Đaković, M. in prepearation.

Keywords: hydrogen bond, halogen bonds, supramolecular synthesis 